MATEC Web of Conferences 16, 03006 (2014)

DOI: $10.1051 /$ matecconf/ 20141603006

(C) Owned by the authors, published by EDP Sciences, 2014

\title{
Nonlinear responses of externally excited rotor bearing system
}

\author{
Balram Choudhary and Barun Pratiher \\ Mechanical Engineering Department, Center for Energy, Indian Institute of Technology Jodhpur, India
}

\begin{abstract}
A mathematical model incorporating higher order deformation in bending is developed to investigate the nonlinear behavior of rotor. Transverse harmonic base excitation is imparted to rotor system and Euler-Bernoulli beam theorem is applied with effects such as rotary inertia, gyroscopic effect, higher order large deformations, rotor mass unbalance and dynamic axial force. Discretization of the kinetic and strain (deformation) energies of the rotor system is done using the Rayleigh-Ritz method. Second order coupled nonlinear differential equations of motion are obtained using Hamilton's principle. Nonlinear dynamic response of the rotor system is obtained by solving above equations using the method of multiple scales. This response is examined for resonant condition. It is concluded that nonlinearity due to higher order deformations and variations in the values of different parameters like mass unbalance and shaft diameter significantly affects the dynamic behavior of the rotor system. It is also observed that the external harmonic excitation greatly affects the dynamic response.
\end{abstract}

\section{Introduction}

Rotating machinery [1-3] such as steam turbines, gas turbines, internal combustion engines, and electric motors, are the most widely used elements in mechanical systems. The prediction and analysis of the dynamic behavior of rotor system are crucial because their rotating components possess unlimited amounts of energy that can be transformed into vibrations. Therefore, studying the dynamic response, both theoretically and experimentally, of the flexible rotor system under various loading conditions would help in understanding and explaining the behaviour of rotor system. To simplify the analysis, researchers often try to use the linear analysis. But, application of nonlinear analysis is sometimes inevitable. Many phenomena should be described with nonlinear equations which are not explainable with linear analysis. The importance of considering the nonlinear effects in the dynamic analysis of rotating equipment has increased so as to obtain more accurate and optimized performance. Nonlinearities in rotor systems can be due to higher order large deformations, rotor-base excitations [3], geometric nonlinearities, oil film in journal bearings, magnetic bearings. The analysis of the nonlinear effects in rotorbearing systems is extremely difficult and there are a few analytical procedures that will generate valid results over a wide range of parameters. A geometrically nonlinear model of a rotating shaft was introduced by Luczko [4]. The model included Von-Karman nonlinearity, nonlinear curvature effects, large displacements and rotations as well as gyroscopic and shear effects. Vibration problems involving nonlinearities do not generally lend themselves to closed form solutions obtained by using conventional analytical techniques. The perturbation methods [5] are a collection of techniques that can be used to simplify, and to solve, a wide variety of mathematical problems, involving small or large parameters. The solutions may often be constructed in explicit analytical form or, when it is impossible, the original equation may be reduced to a simpler one that is much easier to solve numerically.

In this paper, analytical and numerical investigation of rotor system having transverse harmonic excitation is done considering the nonlinearity due to higher order deformation in bending. The restricted axial motion of rotor results in axial dynamic force, causing large deformation in bending. A nonlinear mathematical model has been developed; which includes various secondary effects like rotary inertia effects, gyroscopic effects and rotor mass unbalance. The method of multiple scales [5] is applied is used to solve this model including nonlinear terms. This method is applied directly to the partial differential equation of motion and to the discretized equations. The results of perturbation method are validated with numerical simulations. 


\section{Problem formulation}

The rotor system consists of a flexible shaft and a rigid disk and has the same rotor geometry as used by Duchemin et al. [1] as shown in figure 1. The shaft considered, is a beam of circular cross section of radius $R_{1}$ and length $\mathrm{L}$, and spins about longitudinal axis y with a constant speed $\Omega$. The disk of mass $M_{\mathrm{d}}$, external radius $\mathrm{R}_{2}$ and internal radius $\mathrm{R}_{1}$, is positioned on shaft at a distance $L_{d}$ along the y axis. The mass unbalance denoted by $m_{\mathrm{u}}$ is also situated at a distance, $y=L_{d}$. The shaft is flexible, so it is modeled by its kinetic and strain energies, whereas the rigid disk is modeled by its kinetic energy only. Transverse harmonic base excitation $\mathrm{Q}_{2}=P_{0} \cos \left(\omega_{\mathrm{e}} \mathrm{t}\right)$ is applied to rotor system as shown in Fig. 1.

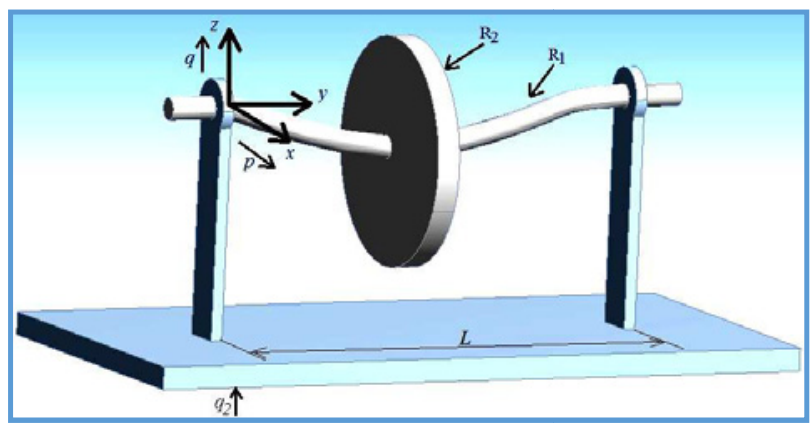

Fig.1. Rotor with shaft and disk

Using extended Hamilton's principle, finally nonlinear structural dynamics of rotor bearing system under harmonic base motion is expressed as [1]

$\ddot{P}-\Omega \vartheta_{1} \dot{Q}+\vartheta_{2} P+\left(\frac{1}{2} \mu_{1}+\mu_{2}\right)\left(P^{3}+P Q^{2}\right)+c \dot{P}=$

$m_{1} \Omega^{2} d_{1} f\left(L_{d}\right) \sin \Omega t$

$\ddot{Q}+\Omega \vartheta_{1} \dot{P}+\vartheta_{2} Q+\left(\frac{1}{2} \mu_{1}+\mu_{2}\right)\left(Q^{3}+P^{2} Q\right)+c \dot{Q}=$

$m_{1} \Omega^{2} d_{1} f\left(L_{d}\right) \sin \Omega t-\gamma \ddot{Q_{2}}$

Here, $P$ and $Q$ are generalized independent coordinates and $f(y)$ is the displacement function and has been considered as the normalized first mode shape of a beam with a constant cross section in bending. $f(y)=$ $\sin (\pi y / L), \quad f^{\prime}(y)=g(y)$, and $\quad f^{\prime \prime}(y)=h(y)$. Following is the expressions for the coefficients in the equations (1-2).

$F_{1}=\operatorname{EI} \int_{0}^{\mathrm{L}} h^{2}(\mathrm{y}) d y, \quad F_{2}=\operatorname{EA} \int_{0}^{\mathrm{L}} g^{4}(\mathrm{y}) d y$,

$a_{1}=\mathrm{M}_{d} f^{2}\left(L_{\mathrm{d}}\right)+\mathrm{I}_{\mathrm{dx}} g^{2}\left(L_{\mathrm{d}}\right)+\rho \mathrm{A} \int_{0}^{\mathrm{L}} f^{2}(y) \mathrm{dy}+$ $\rho \mathrm{I} \int_{0}^{\mathrm{L}} f^{2}(y) \mathrm{dy}, F_{2}=\frac{\mathrm{EA}}{\mathrm{L}} \int_{0}^{L} \int_{0}^{\mathrm{L}} g^{4}(\mathrm{y}) d y d y$,

$a_{2}=\mathrm{I}_{\mathrm{dy}} g^{2}\left(L_{\mathrm{d}}\right)+2 \rho \mathrm{I} \int_{0}^{\mathrm{L}} g^{2}(y) \mathrm{dy}$,

$\mathrm{c}_{1}=2 \mathrm{M}_{d} f\left(L_{d}\right)+2 \rho \mathrm{A} \int_{0}^{\mathrm{L}} f(y) \mathrm{dy}, \vartheta_{1}=b_{2} / b_{1}$,
$\vartheta_{2}=F_{1} / b_{1}, \mu_{1}=F_{2} / b_{1}, \mu_{2}=F_{3} / b_{1}, m_{1}=$

$\mathrm{m}_{\mathrm{u}} / b_{1}$, and $\gamma=c_{1} / b_{1}$.

\section{Perturbation techniques}

By applying the perturbation techniques $[5,6]$ with putting the expressions $\vartheta_{1}=\vartheta_{1}, \vartheta_{2}=\vartheta_{2}, \mu_{1}=$ $\varepsilon \mu_{1}, \mu_{2}=\varepsilon \mu_{2}, m_{1}=\varepsilon m_{1}, c=\varepsilon c, \gamma=\varepsilon \gamma$, one may have the following expressions obtained by equating the coefficients of the like powers of $\varepsilon$ on both sides of the resulting equations.

System of order 0 equations $\left(\varepsilon^{0}\right)$

$\frac{\partial^{2} p}{\partial T_{0}^{2}}+\vartheta_{2} p_{0}-\Omega \vartheta_{1} \frac{\partial q_{0}}{\partial T_{0}}=0$

$\frac{\partial^{2} q_{0}}{\partial T_{0}^{2}}+\vartheta_{2} q_{0}+\Omega \vartheta_{1} \frac{\partial p_{0}}{\partial T_{0}}=0$

System of order 1 equations $\left(\varepsilon^{1}\right)$

$\frac{\partial^{2} p_{1}}{\partial T_{0}^{2}}+\vartheta_{2} p_{1}-\Omega \vartheta_{1} \frac{\partial q_{1}}{\partial T_{0}}=\Omega \vartheta_{1} \frac{\partial q_{0}}{\partial T_{0}}-2 \frac{\partial^{2} p_{0}}{\partial T_{0} \partial T_{1}}-\frac{\mu_{1} p_{0}^{3}}{2}-$

$\frac{\mu_{1} p_{0} q_{0}^{2}}{2}-\mu_{2} p_{0}^{3}-\mu_{2} p_{0} q_{0}^{2}-c \frac{\partial p_{0}}{\partial T_{0}}+$

$m_{1} \Omega^{2} d_{1} f\left(L_{d}\right) \sin \Omega t$

$\frac{\partial^{2} q_{1}}{\partial T_{0}^{2}}+\vartheta_{2} q_{1}+\Omega \vartheta_{1} \frac{\partial p_{1}}{\partial T_{0}}=-\Omega \vartheta_{1} \frac{\partial p_{0}}{\partial T_{0}}-2 \frac{\partial^{2} q_{0}}{\partial T_{0} \partial T_{1}}-\frac{\mu_{1} q_{0}^{3}}{2}-$

$\frac{\mu_{1} q_{0} p_{0}^{2}}{2}-\mu_{2} q_{0}^{3}-\mu_{2} q_{0} p_{0}^{2}-c \frac{\partial q_{0}}{\partial T_{0}}+$

$m_{1} \Omega^{2} d_{1} f\left(L_{d}\right) \cos \Omega t-\gamma \ddot{q}_{2} / 2$

The solution of above equations is given as

$p_{0}=D_{1}\left(T_{1}\right) \exp \left(i \omega_{1} T_{0}\right)+D_{2}\left(T_{1}\right) \exp \left(i \omega_{2} T_{0}\right)+[c c]$

$q_{0}=i D_{1}\left(T_{1}\right) \exp \left(i \omega_{1} T_{0}\right)-i D_{2}\left(T_{1}\right) \exp \left(i \omega_{2} T_{0}\right)+[c c]$

Here. [cc] denotes the complex conjugate. Substitution of equations 8-9 into equations 6-7 and then putting, $\omega=\omega_{1}+\varepsilon \sigma_{1}$ where $\sigma_{1}$ is a detuning parameter for controlling the nearness of $\omega_{\mathrm{e}}$ to $\omega_{1}$, one may obtain the solvability conditions. The first step in determining these solvability conditions is to substitute $\Omega=\omega_{1}+\varepsilon \sigma_{1}$, and equate the coefficients of $\exp \left(i \omega_{1} \mathrm{~T}_{0}\right)$ and $\exp \left(i \omega_{2} \mathrm{~T}_{0}\right)$ on both sides of the resulting particular solution and by following similar procedures in [5,6] with separation of real and imaginary terms, one may obtain, following a set of four first order differential equations

$\frac{1}{2} \frac{\partial d_{2}}{\partial T_{1}}+\frac{1}{2} g_{5} d_{2}=0$

$\frac{1}{2} d_{2} \frac{\partial \theta_{2}}{\partial T_{1}}+\frac{1}{8} g_{2} d_{2}^{3}+\frac{1}{8} g_{3} d_{1}^{2} d_{2}+\frac{1}{2} g_{6} d_{2}=0$

$\frac{1}{2} \frac{\partial d_{1}}{\partial T_{1}}+\frac{1}{2} h_{5} d_{1}+h_{4} \cos (\mu)=0$ 
$-\frac{1}{2} d_{1} \sigma_{1}+\frac{1}{2} d_{1} \frac{\partial \mu}{\partial T_{1}}-\frac{1}{8} h_{2} d_{1}^{3}-\frac{1}{8} h_{3} d_{1} d_{2}^{2}-\frac{1}{2} h_{6} d_{1}-$

$h_{4} \sin (\alpha)=0$

Here, $\alpha=-\theta_{1}+\sigma_{1} T_{1}, g_{2}=-\frac{\left(\beta_{1}+2 \beta_{2}\right)}{\omega_{2}}, g_{3}=2 c_{2}, g_{5}=$ $\frac{c}{2}, g_{6}=\frac{\Omega \alpha_{1}}{2} \quad, \quad h_{2}=-\frac{\left(\beta_{1}+2 \beta_{2}\right)}{\omega_{2}}, h_{3}=2 h_{2}, h_{4}=$ $\frac{\gamma \omega_{e}^{2} P_{0}}{8 \omega_{1}}, h_{5}=\frac{c}{2}$, and $h_{6}=\frac{\Omega \alpha_{1}}{2}$.

\section{Result}

In all the simulations, Euler-Bernoulli beam element with length $\mathrm{L}=0.4 \mathrm{~m}$, mass density $\rho=7800 \mathrm{~kg} / \mathrm{m}^{3}$, Young's modulus $=2 \times 10^{11} \mathrm{~N} \mathrm{~m}^{2}$, mass moment of inertia in $\mathrm{x}$ direction $=0.11 \mathrm{kgm}^{2}$, mass moment of inertia in $y$ direction $=0.19 \mathrm{~kg} \mathrm{~m}^{2}$, cross-sectional area $=3.1 \times 10^{-4} \mathrm{~m}^{2}$, area moment of inertia $=7.854 \times 10^{-9}$, thickness of the disk $=0.03 \mathrm{~m}$, and inner and outer radius of the disk are equal to 0.01 and $0.15 \mathrm{~m}$, respectively has been considered. The solid line represents responses of shaft in the direction $z$ while the dotted line represents the time history of the shaft in the direction $x$ in Fig. 2. Time response has been obtained for $P_{0}$ equal to $0.001 \mathrm{~m}$ and $m_{\mathrm{u}}$ equal to $0.01 \mathrm{~kg}$ when other parameters are keeping constant. From the Fig. 2, it has been observed that amplitude for the response $(Q)$ in transverse direction is higher than the response $(P)$ amplitude generally occurred in $x$ direction. Hence, rotor bearing system is more stiff in $x y$ plane than in the plane perpendicular to the $x y$ i.e., in $z$ direction. One more observation has been noticed that while time response in the transverse direction shows a quasiperiodic in nature, a beating phenomenon is observed in the time history representing the response in $x$ direction as forcing frequency is closed to first natural frequency of the shaft in $x$ direction.

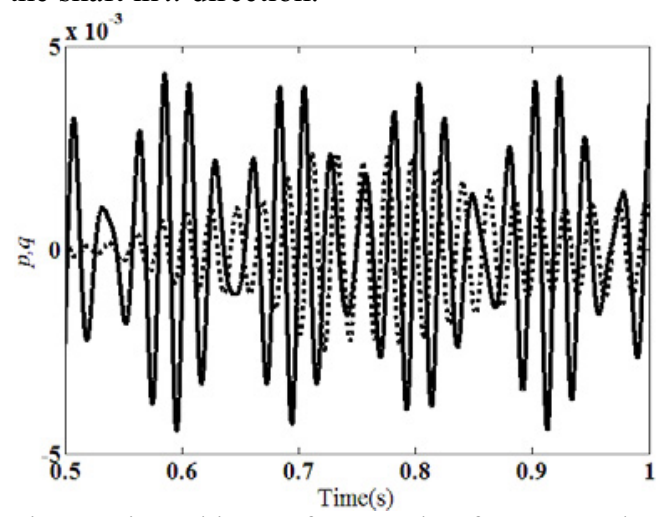

Fig. 2: Times history for $P$ and $Q$ for $P_{0}$ equal to 0.001 .

Figure 3 and 4 illustrate the effect of amplitude of external forcing stimulated due to support motion for $P_{0}$ equal to 0.001 and 0.01 on the dynamics performance of the rotor bearing system. It has been observed that as expected with increase in amplitude of base motion, the amplitude gets increased for the responses of both directions. However, response amplitude of response in transverse direction is more eminent over the amplitude in other directions though it has been considered that the elastic deformation of the shaft is constrained in the $y$ direction.

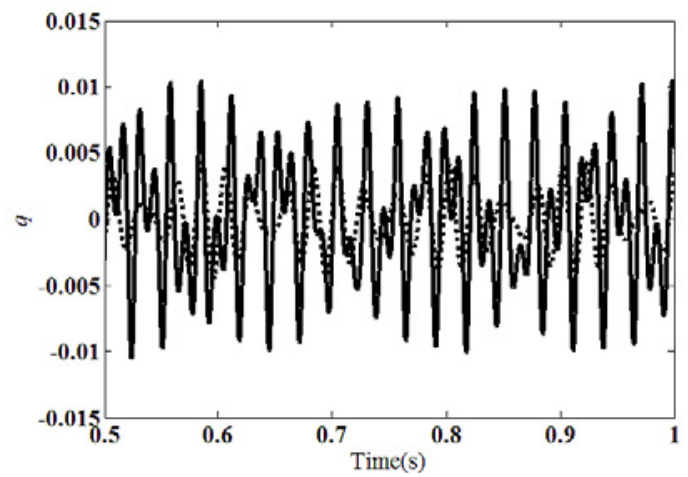

Fig. 3: Time history for $\mathrm{Q}$ for $P_{0}$ equal to 0.001 and 0.01 .

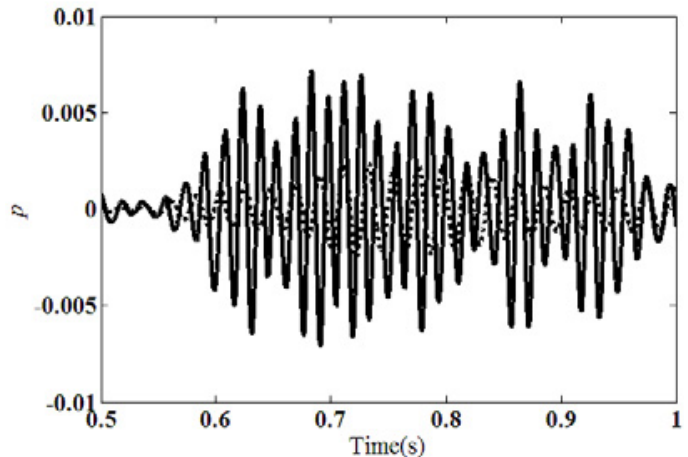

Fig. 4: Time history for $P$ for $P_{0}$ equal to 0.001 and 0.01 .

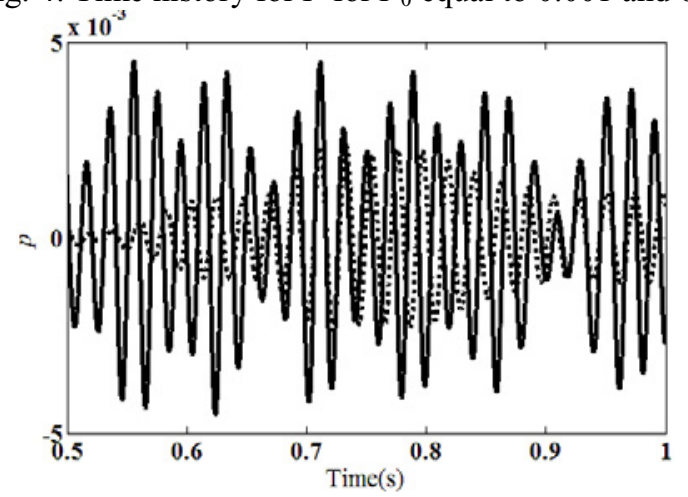

Fig. 5: Effect of mass imbalance on responses in $x$ direction

Figure 5 and 6 illustrate the influence of mass imbalance $\mathrm{m}_{\mathrm{u}}$ equal to 0.01 and 0.1 on the dynamics behaviour of the rotor bearing system. While with increase in mass imbalance, the amplitude of responses in transverse direction remains constant although amplitude gets increased for the response in $x$ direction. Figure 7 depicts a comparison study between the response obtained using temporal equation of motion and the response obtained using first order method of multiple scale method. It has been observed that for both responses, the maximum 
amplitude remains the same excluding that the first order solution illustrates a pure periodic solution with equilibrium point of $0.005 \mathrm{~m}$ whereas temporal solution clearly portrays a quasi-periodic solution and vibrates with zero reference. Fig. 8 obtained numerically the temporal equation of motion and reduced first order set of algebraic equations (12)-(13).

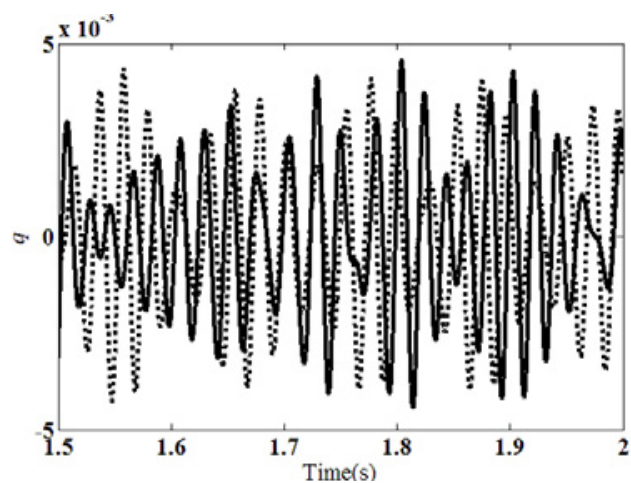

Fig. 6: Effect of mass imbalance on responses in transverse direction

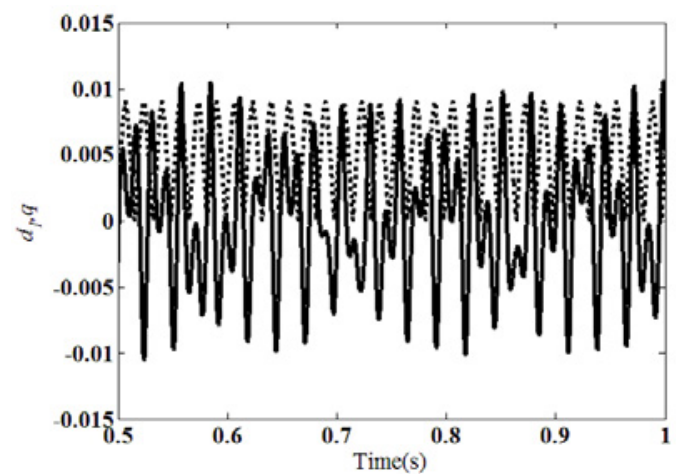

Fig. 7: Time history for temporal solution and first order solution (dotted line).

\section{Conclusion}

Nonlinear dynamic response of the rotor system is obtained by solving above equations using the method of multiple scales. This response is examined for resonant condition when the forcing frequency is nearly equal to the first natural frequency of shaft in $x$ direction. It is concluded that nonlinearity due to higher order deformations and variations in the values of different parameters like mass unbalance and shaft diameter significantly affects the dynamic behavior of the rotor system. It is also observed that the external harmonic excitation greatly affects the dynamic response
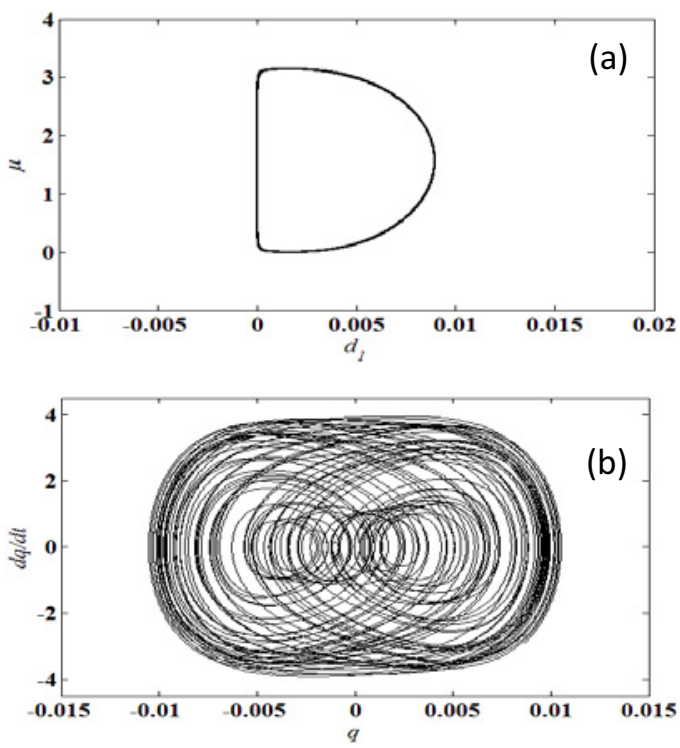

Fig. 8: Phase portrait a. reduced equations 12-13 b. temporal equations 1-2.

\section{References}

1. S. Rizwan, M. Guilhem, B. Alain. Modeling and analysis of nonlinear rotordynamics due to higher order deformations in bending, Applied Mathematical Modelling 35( 5) 2145-2159 (2011)..

2. L.M. Adams, J.R. Adama, Rotating Machinery Vibrations from Analysis to Troubleshooting, Dekker, New York, 2001.

3. M. Duchemin, A. Berlioz, G. Ferraris, Dynamic behavior and stability of a rotor under base excitations, J. Vibration and Acoustics, 128(5) 576585 (2006).

4. J. Luczko, A geometrically nonlinear model of rotating shafts with internal resonance and self excited vibrations, J. Sound and Vibrations, 255(3) 433-456 (2002).

5. A.H. Nayfeh, Introduction to Perturbation Techniques, Wiley, New York, 1993.

6. S. Hosseini, S. Khadem, Free vibration analysis of a rotating shaft with nonlinearities in curvature and inertia, Mechanism and Machine theory, 44, 272-288 (2009).

7. H. Yabuno, Y. Kunitho, T. Inoue, Y. Ishida, Nonlinear analysis of rotor dynamics by using method of multiple scales, Iutam Symposium on Dynamics and Control of Nonlinear Systems with Uncertainty 2, 167-176 (2007). 